\section{DESCONCIERTO DEL TIEMPO PRESENTE Y RETOS DEL FUTURO. ALGUNAS CLAVES ORIENTATIVAS}

\author{
Manuel González Portilla \\ José Urrutikoetxea Lizárraga \\ Miembros del Grupo de Demografía Histórica e Historia Urbana \\ Departamento de Historia Contemporánea/ \\ Gauregungo Historia Saila, UPV/EHU
}

\section{UNCERTAINTY OF PRESENT TIMES AND FUTURE CHALLENGES. SOME KEY ASPECTS}

\begin{abstract}
The uncertainty is one of the most appropriate characteristics of our west society. This has been in the forefront of the world for centuries, but now his protagonist role is decreasing, so his next future is under question. The recent history illuminates some key points: the irruption of the great demographic powers -China and India-, the acceleration of the industrialisation, the world-wide urban development, and the swing of the economic core towards the Pacific area. Other changes in process actually are: the demographic transition (mortality and fecundity), the change in the values and the family patterns, the influence of the new economy based on the knowledge and the innovation, and the growing role of the women in all the society fields.
\end{abstract}

KEY WORDS: Present world; crisis; urbanization; industrialisation; demographic transition; development geography; innovation; knowledge economy; women.

\begin{abstract}
RESUMEN: La de la incertidumbre es una de las caracteristicas que mejor define a nuestra sociedad occidental. Vanguardia del mundo durante siglos, se encuentra con que ve cómo se recorta su protagonismo. Desde esa incertidumbre, se cuestiona por su futuro inmediato. La historia reciente pone ante nuestros ojos algunas claves: aparición en escena de grandes potencias demográficas (China e India), industrialización, desarrollo urbano mundial y desplazamiento hacia el Pacífico del eje económico planetario son tres de las más evidentes. En un plano acaso no tan evidente, se están produciendo otros cambios de enorme calado: transición demográfica (mortalidad y fecundidad), cambios en los valores y tipologías familiares, papel insoslayable de una economía basada en la innovación y el conocimiento $(I+D+i)$ y presencia creciente e insustituible de la mujer en todos los ámbitos de la sociedad.
\end{abstract}

PALABRAS CLAVE: Mundo presente; crisis; urbanización; industrialización; transición demográfica; ejes mundiales; innovación; economia del conocimiento; mujer.

"El hombre ha sobrevivido hasta ahora porque era demasiado ignorante para cumplir sus deseos. Ahora que ya puede cumplirlos, tiene que cambiarlos o perecer"

(W. C. WiLLIAMS, 1952)

"Intelectual o no, ningún ser humano que viva en el Occidente de fines del siglo XX escapa a esta angustia de la pérdida de todo sentido"

(A. Tourain, 1993)

\section{INTRODUCCIÓN}

Los grandes cambios de los dos últimos siglos indican que algo significativo está ocurriendo en la historia de la Humanidad. Nunca el proceso histórico ha conocido una aceleración tan intensa. Conviene subrayar, en todo caso, que se trata de un proceso protagonizado por un conjunto muy reducido de generaciones. Son las últimas ocho o diez las que han impulsado el cambio más radical que ha experimentado la humanidad en los últimos diez 
mil años, si bien la verdadera intensificación de estos ritmos ha corrido básicamente a cargo de las cuatro últimas. $Y$ todo indica que en un plazo de tiempo breve, quizás cuatro generaciones, puede poner ante nosotros una realidad profundamente distinta de la actual. Para ese momento, casi toda la humanidad vivirá previsiblemente inmersa en esta nueva dinámica. Se trata de un camino sin retorno.

Durante mucho tiempo la historiografía asistió dubitativa al debate sobre lo adecuado o no de incluir dentro de su ámbito de reflexión los problemas históricos más inmediatos. Hace ya unas décadas que superó esa timidez, de manera que actualmente se trata de una opción epistemológica aceptada. Hoy día, disponemos ya de herramientas y conocimientos lo suficientemente sólidos como para intuir por dónde va a ir la historia del futuro más cercano, básicamente el de las dos próximas generaciones. Es este convencimiento el que nos anima a tratar de sacar a la luz algunas de las líneas de fuerza de larga duración que se van dejando entrever, no tanto en los acontecimientos puntuales o en los discursos, sino en los procesos históricos de fondo que caracterizan a las sociedades del presente. El repaso de algunos de esos paradigmas puede ayudar a localizar y comprender el significado de la aceleración de este proceso histórico.

Esta reflexión sobre las conexiones entre pasado y futuro la planteamos desde el análisis de tres grandes líneas transformadoras de la realidad histórica contemporánea: la industrialización, la población y el espacio urbano. El estado de conocimiento de que disponemos sobre estas dos últimas permite ya crear modelos bastante certeros respecto de su posible evolución en un plazo de, al menos, cincuenta años. Podemos prever bastante fehacientemente la evolución demográfica y su estructura hasta el 2050. Y lo mismo se puede decir del desarrollo urbano. Sin embargo, la ciencia económica se muestra incapaz de ofrecernos una visión medianamente clara de futuro. No disponemos de herramientas que nos permitan vislumbrar cuál va a ser la estructura productiva futura. A día de hoy, únicamente disponemos de unas pocas certezas: la de que el fututo está vinculado a la llamada economía del conocimiento $y$, por lo tanto, a la producción de bienes y servicios con altos componentes de conocimiento en sus costes finales o la de que el centro económico mundial se situará en el este y sureste asiáticos.

\section{LOS TERRENOS DE LA ECONOMÍA: LA INDUSTRIALIZACIÓN}

Uno de los paradigmas de nuestro modelo de crecimiento bisecular se refiere al tránsito desde una economía básicamente sustentada en la agricultura hasta otra de cuño moderno a la que los estudiosos han identificado como "industrialización". Mientras que la civilización agraria surgió de forma independiente en distintas regiones del mundo, la regida por la industrialización impide, debido al ritmo vertiginoso de su expansión, el nacimiento de sociedades industriales por vías independientes. El modelo es, por tanto, único.

Nace en la Europa del noroeste (Inglaterra) en el siglo XVIII y se extiende progresivamente, durante el siglo XIX, por la cuenca del Atlántico Norte (Europa Occidental y del Norte, América del Norte). Durante el último tercio de ese mismo siglo, penetra con fuerza por el resto de Europa y el sur de América para tocar en Asia a través de Japón. Bastante más tarde, ya en la segunda mitad del siglo XX, el modelo avanza con fuerza por el este y sureste asiáticos, implicando a casi todas la naciones de la zona. Entre ellas destacan China e India, hasta el punto de que la cuenca Pacífico-Índico apunta con suplantar la primacía del eje atlántico y convertirse en la protagonista del nuevo modelo de crecimiento.

\subsection{Las bases del modelo}

Nueva división internacional del trabajo

Esta "nueva" economía industrial se expande, ya desde su origen, a lo largo y ancho de un mercado progresivamente más globalizado al que impone nuevas reglas. Establece una nueva división internacional del trabajo. Los países tempranamente industrializados exportan manufacturas, bienes industriales, capitales y personas, mientras que las zonas más atrasadas y menos industriales son exportadoras de materias primas (alimentos y minerales, fundamentalmente) e importadoras de manufacturas, capitales y personas provenientes de los países industriales. Esta nueva división internacional del trabajo da origen a un nuevo período de relaciones internacionales. El colonialismo y el imperialismo de las potencias industriales imponen un nuevo orden. En el momento del inicio de la Primera Guerra Mundial (1914), más del 80\% del planeta está gestionado por las potencias industriales del llamado "Mundo Occidental". 
Nuevo sistema productivo y social

En su base se encuentra un nuevo sistema socio-productivo: el capitalismo. El mercado se convierte en el motor fundamental de las reglas de juego del sistema, y éstas afectan directamente, entre otros, al factor trabajo. La nueva sociedad pasa a articularse en torno a clases trabajadoras, clases medias y elites económicas. Entre las primeras, el proletariado juega un papel significativo. Las clases medias alcanzan una presencia creciente, en tanto que empresarios y burgueses dan imagen y contenido social a las nuevas elites. Por el contrario, el intenso repliegue del mundo campesino dibuja la otra cara de este proceso. La población activa agraria de los paises industriales pasa del $80 \%$ de inicios del siglo XIX a menos del 5\% de finales del XX.

\section{Aceleración temporal y proximidad espacial}

La reiterada referencia a generaciones trata de situar a los lectores ante una realidad cercana, pero intensamente cambiante: ellos mismos y sus antepasados más inmediatos son los verdaderos protagonistas de este cambio. Como modelo económico dominante, la civilización agraria se prolongó por cerca de 10.000 años. Esto, traducido en experiencia humana, equivale a cuatrocientas generaciones. Aunque los cambios tecnológicos y productivos a lo largo de estos 10.000 años fueron significativos, sin embargo, las técnicas de cultivo, la alimentación y las formas de vida se modificaron muy lentamente. Generaciones sucesivas, calculadas en decenas, apenas eran capaces de percibir estos cambios. Aparentemente, similares procesos se repetían generación tras generación. Por el contrario, la percepción que, a comienzos del siglo XXI, tiene un individuo del tiempo histórico y del espacio es radicalmente distinta de la que tenían sus padres, abuelos o bisabuelos. Y no es sólo eso; tememos, además, ser arrollados por un futuro inmediato que se nos antoja enigmático.

Por otro lado, todo nos resulta cada vez más cercano. Viajamos a diversos continentes en muy pocas horas y conocemos múltiples paises y ciudades. Mientras que, para la generación de nuestros abuelos, lo cercano ero lo determinante y relevante, para nosotros la realidad vital se globaliza, incluso en términos de trabajo. Acontecimientos que suceden en otras partes del mundo inciden sobremanera en nuestra vida cotidiana. Es el caso de la crisis financiera nacida en Estados Unidos en 2007-2008, la entrada de decenas de millones de trabajadores en las nuevas industrias manufactureras chinas, la consiguiente desaparición de miles de industrias occidentales o los bajos costes de la mano de obra china que permiten aumentar el consumo de manufacturas y el ahorro relativo de las familias occidentales. Las tecnologías relacionadas con la comunicación están ampliando los procesos de comunicación y conocimiento a escala mundial. La frecuencia de las relaciones y el volumen de la información de la que disponemos son prácticamente inabarcables. Se calcula que, en 2008, se produjeron unos 1.200 exabytes de información en la red (Un exabyte es igual a 2 elevado a 60)'.

Cabe resumir lo dicho hasta aquí señalando que, a lo largo de los últimos dos siglos y medio, la nueva economía industrial ha actuado como matriz en torno a la cual se articulan todos estos grandes cambios. No sólo propicia un aumento sustancial de la producción, sino que, a su vez, conlleva un incremento de la productividad. Ello deriva, directamente, en un crecimiento de los recursos disponibles por habitante, todo ello dentro de una dinámica global que se va acelerando a medida que nos acercamos al momento actual y que nos ha llevado a interiorizar un modelo de desarrollo basado en una presunción hoy profundamente debatida: la del crecimiento continuo e ilimitado.

\subsection{Las grandes fases del proceso industrializador: de la "innovación tecnológica" a la "revolución científica"}

Conviene establecer una primera divisoria de partida en el seno de toda esta larga historia. La primera fase de este proceso industrializador se prolonga desde sus inicios en 1780 hasta 1880. En ella, los ciclos de innovación se vinculan básicamente a los avances tecnológicos. Marcha de la mano del nacimiento del Estado-nación y del desarrollo de un capitalismo que evoluciona de los principios y prácticas más liberales a otros de carácter monopolista, colonial e imperialista y asiste a la aparición en la escena social de nuevas clases. La segunda fase se corresponde básicamente con la mayor parte del siglo XX y vincula su crecimiento, también a los avances tecnológicos, pero sobre todo a la impronta que marcan los avances científicos ("revolución científica") y al cumplimiento íntegro de la "transición demográfica" con el control de la fecundidad. 


\subsubsection{Primera Industrialización y avances tecnológicos (1780-1880)}

La primera fase de la industrialización está estrechamente vinculada a los cambios tecnológicos y a los sucesivos ciclos de innovación que la componen.

Primer ciclo (1780-1830). El inicio del gran salto.

De INGLATERRA AL OCCIDENTE NORATLÁNTICO.

ALGODÓN, MÁQUINA DE VAPOR Y HIERRO

El primer ciclo, iniciado en el último tercio del siglo XVIII, está vinculado a la revolución industrial inglesa. Sus innovaciones más significativas marchan de la mano de la máquina de vapor y de su aplicación a las máquinas de hilar y tejer, a la minería y a otros procesos productivos. Las nuevas materias primas que se utilizan son el algodón, en la producción textil, y el coque como nuevo combustible utilizado en la producción de hierro. El uso industrial del algodón permite romper el cuello de botella que, en la fabricación de tejidos, suponía una producción de lana limitada por la cantidad de ovejas y por la extensión de tierra. El coque, por su parte, sustituye al carbón vegetal. La deforestación había avanzado sustancialmente en el mundo euroasiático y era especialmente llamativa en la Inglaterra de finales del XVII.

Este primer ciclo pone los cimientos de la nueva economía. La producción en masa y para las masas permite que los costes y los precios por unidad de producción desciendan a largo plazo. En consecuencia, porcentajes cada vez mayores de población van a poder comprar más productos (tejidos, herramientas, etc.). Para finales del primer tercio del siglo XIX, Inglaterra se ha convertido en la gran fábrica del mundo.

Segundo ciclo (1830-1880). EXPANsión del Modelo OCCIDENTAL. Europa, Estados Unidos y Japón. Transporte y acero

Este segundo ciclo de innovaciones está marcado igualmente por los sucesivos avances tecnológicos desarrollados a partir del primer ciclo. Entre aquéllas y de acuerdo con la relevancia que van a tener en el desarrollo económico posterior, destacamos la aplicación de la energía de vapor y de la máquina de vapor al transporte y a la producción de acero por vía directa. La aparición del ferrocarril y de la navegación a vapor hace que se conozca al período como el de la revolución del transporte. En su faceta terrestre, el ferrocarril posibilita mover grandes cantidades de mercancía a largas distancias y a muy notable velocidad. El salto es enorme comparado con el transporte a "uña y sangre" hegemónico hasta ese momento. Por su parte, la producción de acero experimenta un cambio radical gracias al avance que supone la simplificación de su producción. A través de un único proceso desarrollado en un horno convertidor (Bessemer, Martin, Siemens, Robert, etc.), el arrabio que sale del alto horno se transforma en acero. Por primera vez se puede producir acero, por vía directa, en grandes cantidades y a bajos precios. Con ser de crucial importancia, transporte y acero no son, las únicas novedades destacables de este segundo ciclo. Se producen otras como la dinamita, la vulcanización del caucho, el telégrafo, la fotografía, la máquina de escribir, etc.

Este segundo ciclo de innovaciones amplía la geografía de los paises industrializados, tanto en Europa como en América del Norte. Inicia, incluso, su penetración en Asia de la mano de Japón. Para una recta comprensión de lo que significa la transformación que experimenta la economía industrial a lo largo de estos dos ciclos, es preciso señalar, sin embargo, que todavía, a finales del primero de ellos, el peso de la producción industrial de Asia era muy superior al de la europea. Hacia 1830, China e IndiaPakistán alcanzan casi la mitad de la producción mundial (47\%), en tanto que el Occidente no pasa del 31\%. Pues bien; cincuenta años después, a finales del segundo ciclo de innovaciones, el avance de la producción industrial occidental es espectacular. Hacia 1880, procedía de él el $69 \%$ de la producción industrial mundial, mientras que la de China e India-Pakistán caía al 15\% (Bairoch, 1982). En tan sólo medio siglo, el vuelco en las hegemonías productivas mundiales es total. $Y$ ello ocurre prácticamente ante nuestros ojos, en las vísperas del siglo XX.

Pero los cambios no se limitan al solo ámbito de la economía. Simultáneamente van surgiendo nuevas realidades sociales con el complejo entramado de las nuevas instituciones político-administrativas vinculadas al Estado-nación. Nacen sociedades más abiertas y complejas y redes comerciales cada vez más extensas. Los estados nacionales comienzan a desempeñar un rol de primera magnitud. Las ventajas que adquieren los paises occidentales reconfiguran y jerarquizan el panorama económico y político mundial desde los distintos modelos de capitalismo. En la medida en que sus mercados 
no son capaces de absorber su producción, las economías nacionales se encontrarán en la necesidad de hacerse con nuevos mercados, nuevos consumidores y materias primas de todo tipo a bajo precio. Contarán, para ello, con el apoyo del aparato estatal a través de la colonización de amplios territorios a escala mundial y de la explotación de sus recursos humanos y materiales. La Conferencia de Berlín consagrará este nuevo orden. El principio del "hinterland" rubrica la hegemonía occidental y bendice como económica y políticamente correcto este salto hacia delante. Por otro lado, la competencia interna conduce a la empresa capitalista a un proceso de fusiones que le permitirá controlar los mercados nacionales a través de la implantación de prácticas y precios monopolistas. El paso de un primer capitalismo de corte liberal y doctrinario a otro de carácter monopolista, imperialista y colonial es un hecho. Estas prácticas expansivas vienen, naturalmente, arropadas por nuevos discursos ideológicos. El darwinismo social desempeñará una función de primera magnitud en la construcción del pensamiento de las décadas a caballo entre los siglos XIX y XX.

Al mismo tiempo, éste es el momento de la clase obrera. Ésta comienza a manifestar su presencia reivindicativa a través de la creación de sindicatos y partidos de clase frente al capital, lo que le permite obtener avances sustanciales: mejores niveles salariales, descenso de la jornada laboral, logro de seguros sociales relacionados con enfermedades y accidentes, retiro, regulación del trabajo de mujeres y niños, etc. Paralelamente, las clases medias están conformando instituciones básicas para la articulación de la nueva sociedad: nuevas formas y estructuras familiares, impulso y fortalecimiento del estado de derecho, etc. Es ahora cuando nace el sistema de partidos políticos vinculados a los intereses de las clases sociales ascendentes. Ello fortalece el avance paulatino de la democratización del poder politico, desde los modelos de raíz censitaria, pasando por los de marchamo democrático, y que, en determinados casos, desembocan en experiencias socialistas (Revoluciones de 1848) e incluso anarquistas (Comuna de París de 1876). La globalización se sigue extendiendo, esta vez bajo el patrón y modelo de la llamada "civilización occidental".

\subsubsection{Segunda Industrialización y "Revolución científica" (1880-2010)}

Esta segunda fase de la industrialización, que se corresponde prácticamente con el siglo XX, queda definida, más por los avances científicos ("Revolución científica") que por los tecnológicos. Estos últimos, presentes también en esta segunda fase, están cada vez más relacionados con aquéllos. La ciencia se convierte en el motor del nuevo crecimiento económico y de la nueva industrialización. La producción se acelera y lo hacen las tasas de crecimiento a escala mundial, incomparablemente superiores a las de la primera fase.

TeRCER CiClo (1880-1945). CULminación DEL MODELO OCCIDENTAL y CAMBiO de hegemonía. CAPITAL humano, CienCia E InNOVACIÓN

El tercer ciclo de innovación, primero de esta Segunda Industrialización, está vinculado a la producción de mercancías cada vez más complejas. Surgen nuevas fuentes de energía (petróleo y electricidad) y nuevos motores (el de cuatro tiempos, el diesel y el eléctrico) que continúan revolucionando el transporte y otros sectores de servicios. Automóviles, camiones y autobuses desempeñan un papel primordial en un transporte terrestre vinculado a la expansión de nuevas infraestructuras. $Y$ cabe decir otro tanto de la aviación. El automóvil se convierte en el transporte individual frente al trasporte colectivo. El cambio facilita, es verdad, la autonomía individual de los desplazamientos, pero impulsa, al mismo tiempo, la aparición de crecimientos urbanos horizontales comarcalmente especializados a costa de un desmedido gasto en infraestructuras de todo tipo y de un consumo abusivo, radicalmente desigual y no sostenible de energía de origen fósil. Por otra parte, la ciencia y la ingeniería resuelven el dilema del transporte de la electricidad a largas distancias entre centros productores (centrales hidroeléctricas y térmicas) y consumidores (ciudades e industrias).

Electricidad y motor eléctrico van a impulsar el crecimiento de nuevos sectores como el de los electrodomésticos. En su vertiente cotidiana, suponen la tecnificación del hogar y de las labores tradicionalmente consideradas como femeninas. Su implantación progresiva genera espacios crecientes de tiempo libre para la mujer, que paulatinamente irá utilizando parte de este tiempo en la realización de otras actividades distintas de las atribuidas al "ama de casa". Como se verá más adelante, su educación irá ocupando edades y espacios más amplios, que tendrán un notable efecto cultural y social.

Vinculados a este tercer ciclo se desarrollan nuevos sectores productivos: la nueva minería, especialmente la relacionada con el petróleo y sus industrias de transformación (refinerías, etc.) y una potente industria naval cada vez más 
especializada (barcos mineros y petroleros, mercantes y de pasajeros, etc.). Los avances de la química traen consigo la aparición de nuevos productos, fertilizantes y plaguicidas, que impulsan una poderosa revolución verde, con un crecimiento de la producción agraria y de alimentos cada vez más baratos. En términos globales, la humanidad va a disponer cada vez de más recursos alimenticios per cápita. Ahora bien, este crecimiento se va a topar con graves retos: deforestación galopante, transgénicos o agresión a la biodiversidad.

Durante este ciclo de innovaciones, las mercancías producidas son cada vez más complejas y están producidas a partir de muchos componentes que deben acoplase perfectamente para producir esa mercancía final. Esto representa un salto cualitativo en la organización de la producción y del trabajo en los que los criterios de la calidad de los componentes y del proceso productivo son más tenidos en cuenta. El ritmo y el tiempo de la producción en serie desempeñan, por su parte, un papel cada vez más significativo. Surgen y se desarrollan el concepto y la práctica de la organización científica del trabajo (taylorismo y fordismo) y los sistemas de calidad total.

La geografía de este tercer ciclo sigue centrada en un mundo occidental que continúa aumentando su poder a escala mundial. En 1928, aporta el 84\% de la producción industrial mundial. La participación de China e India-Pakistán ha caído hasta el 5\%. No estará de más el recordar que, tan sólo un siglo antes, la producción industrial de China e India-Pakistán alcanzaba en torno al 47\%, en tanto que la occidental no pasaba del $32 \%$. Sin embargo, esta hegemonía occidental va a generar fuertes tensiones internas entre las potencias, lo que se sustancia, al final, con la mayor crisis que viven el capitalismo y las potencias que lo lideran: las dos Guerras Mundiales y la Gran Depresión. La implosión de entre 1914 y 1945 pone punto final a un modelo de desarrollo del capitalismo.
La salida de la crisis se va a efectuar sobre nuevos paradigmas: creciente protagonismo del estado (políticas keynesianas), descolonización y hegemonía de EE.UU. como nueva potencia. El grave debilitamiento que experimentan durante estos años el liberalismo y las ideas democráticas se manifiesta en el fortalecimiento de dos posicionamientos ideológicos antagónicos de los que participan grandes masas de la población y que implican a clases trabajadoras, clases medias y elites. Unas lo hacen en torno a las ideologías socialistas, socialdemócratas, comunistas y anarquistas. Otras, en el círculo de las ideologías totalitarias de derechas: nazismo y fascismo.

Cuarto ciclo (1945-1973/78). "Etapa dorada del crecimiento", hegemonía bipolar y afloración asiática. CAPITAL humano Y MUJER

El cuarto ciclo se inicia al terminar la Segunda Guerra Mundial. Con él se abre un nuevo período definido, en términos económicos, como la "etapa dorada del crecimiento". En términos geopolíticos quedará caracterizado por la hegemonía de las dos superpotencias (EE.UU. y la URSS), la división del mundo en dos grandes bloques y la Guerra Fría. El salto a la "guerra caliente" hubiese supuesto la destrucción de los dos contendientes y sus aliados y, posiblemente, de la humanidad. Período de descolonización masiva y del nacimiento de nuevos estados-nación, es el escenario temporal de un nuevo orden político y económico que se articula en torno a nuevas instituciones: ONU, acuerdos monetarios y financieros nacidos de Bretton Woods (1944), FMI, Banco Mundial o el GATT (Acuerdo General sobre Tarifas Aduaneras y Comercio). Estados Unidos se convierte en la gran potencia del mundo. En 1953, su producción industrial supone el 45\% del total mundial. Las ayudas a Europa a través del Plan Marshall permiten que ésta entre en una intensa etapa de crecimiento económico.

TABLA 1. PARTICIPACIÓN EN LA PRODUCCIÓN INDUSTRIAL MUNDIAL (\%)

\begin{tabular}{|c|c|c|c|c|c|}
\hline & 1830 & 1880 & 1913 & 1953 & 1980 \\
\hline Reino Unido & 9,8 & 22,8 & 13,6 & 8,4 & 4,0 \\
\hline Estados Unidos . .............. & 2,7 & 14,7 & 31,9 & 44,7 & 31,5 \\
\hline China+India-Pakistán $\ldots \ldots \ldots \ldots$ & 47,8 & 15,3 & 4,9 & 4,0 & 7,3 \\
\hline TOTAL & 100 & 100 & 100 & 100 & 100 \\
\hline
\end{tabular}

Fuente: Bairoch, 1982 
Una parte sustancial de este crecimiento económico se basa en el desarrollo masivo de innovaciones producidas en los ciclos anteriores (acero, automóvil o electrodomésticos), la generalización de infraestructuras diversas, el uso masivo de materias primas ya conocidas y de fuentes energéticas fósiles (carbón, petróleo y gas). Por su parte, las innovaciones específicas de este nuevo ciclo están relacionadas con una fuente energética nueva, la atómica, el desarrollo de la química y la farmacopea: antibióticos, vacunas, medios contraceptivos ("píldora"), etc. La carrera por la conquista del espacio y la armamentista potencian innovaciones y productos como los relacionados con la electrónica, la balística, el espacio exterior, la navegación aérea y las telecomunicaciones. Los medios de comunicación de masas alcanzan un protagonismo acrecentado y su difusión se generaliza debido a su bajo precio.

La ciencia continúa su expansión y su creciente diversificación con una difusión cada vez mayor, gracias al acortamiento de tiempos y distancias, lo que incrementa la intercomunicación de los conocimientos a través de congresos, seminarios, revistas científicas especializadas, etc. El desarrollo educativo de los paises industrializados alcanza ahora cotas casi universales. Este asentamiento de los avances educativos y su traducción en cotas más elevadas de productividad tienen traducciones de orden muy plural en los paises industrializados. Los costes de los productos, a precios constantes, siguen disminuyendo con lo que continúa aumentando la capacidad de compra de la población.

El cambio educacional se traduce en cambio social, político y cultural, lo que alcanza una repercusión significativa en el mundo femenino. La mujer de los paises desarrollados -progresivamente y de manera mimética, la de los países que se aproximan a su modelo-, asume activamente el protagonismo de su propia vida y hace escuchar paulatinamente su voz en los ámbitos de "la alcoba", de la educación y de la salud (H. Rosling, 2010). La transición de los modelos de maternidad y de fecundidad, la incursión generalizada, aunque todavía minorizada, de la mujer en el ámbito laboral asalariado y su presencia en la actividad social, cultural, educativa y política comienzan a suponer un cambio radical en los patrones de comportamiento del mundo desarrollado, un cambio que, independientemente de contextos culturales o religiosos, marca unas pautas de desenvolvimiento progresivamente comunes que van de los hechos a los hábitos y de éstos a los valores y a los principios. Y no se olvide; estamos ante la historia de tan sólo tres o cuatro generaciones de mujeres, pero, al mismo tiempo, ante la intrahistoria de un cambio general y radical del que apenas si intuimos sus inicios.

Este cuarto ciclo es impensable, por otro lado, sin la emergencia y el reforzamiento del Estado que, en su forma benéfica, se traduce en el "Estado de bienestar". Gran dinamizador de este proceso y dotado de unos recursos y capacidades sin precedentes, desarrolla sectores que consumen grandes recursos: infraestructuras de todo tipo, educación, sanidad o sistema de pensiones universal (T. Judt, 2008).

Al igual que ocurria en ciclos anteriores, también éste asiste a un claro cambio de coordenadas. La industrialización se abre a nuevas geografías: la del amplio marco que representan la URSS y el bloque del Este, pero, sobre todo, aquella otra que, más allá del caso japonés, se identifica con los inicios del despegue industrial del este y sudeste asiáticos: los "tigres asiáticos" (Hong Kong, Corea del Sur, Taiwán y Singapur).

\section{Quinto ciclo (1973/78-2010). Del Atlántico al Pacífico: \\ China, India, América. Nuevas economias y nuevo reparto de las hegemonias industriales. El retorno de Asia}

La crisis del petróleo y de materias primas (1973-78) abre una nueva etapa. La creación del "Estado del bienestar" y las políticas keynesianas del final del ciclo expansivo de la postguerra generan tensiones inflacionistas y crecientes déficits públicos difíciles de afrontar con la nueva situación de crisis. Los ajustes llegan de la mano de las políticas neoliberales (Reagan y Thatcher) que se van imponiendo en las décadas siguientes ante el desánimo, dejación o renuncia de los ideales y de los proyectos progresistas que alentaron parte importante de los logros anteriores (T. Judt, 2008 y 2010). El nuevo ciclo expansivo se sustentará, en adelante, en una nueva política económica que supone un giro sustancial en la construcción del modelo del "Estado de bienestar" y en los procesos de distribución de las rentas. Frente a la mejora progresiva que experimenta el reparto de la riqueza a lo largo del siglo XX con una creciente mejora para las clases trabajadoras, el "índice Gini"2 pone de manifiesto cómo, en los últimos decenios, se produce una regresión con una polarización de su distribución. Entre 1970 y 2006, en EE.UU. este índice pasa 
del nivel 39 al 47, tendencia que se constata igualmente en la mayoría de los países de OCDE.

En todo caso y hasta hace muy escasos años, los datos del crecimiento económico del mundo occidental seguian siendo importantes. Por si fuera poco, el éxito económicocientífico del mundo occidental dejaba fuera de juego en la geopolítica mundial a la URSS y al bloque socialista que, en los años ochenta y noventa, entran en un rápido proceso de descomposición. La ausencia de otros referentes sólidos hace que este bloque trate de reestructurarse en torno a los parámetros de todo signo y naturaleza del neoliberalismo imperante en el mundo capitalista y occidental.

La industrialización, entendida como nueva civilización distinta de la agraria, continúa su expansión y alcanza un enorme éxito en los países asiáticos más orientales. Durante el ciclo expansivo anterior (1945-73), Japón mantuvo una tasa de crecimiento de en torno al 10\% anual. El modelo se extiende y refuerza en los "Tigres asiáticos" que mantienen elevadas tasas de crecimiento desde la década de los sesenta hasta el momento actual. Pero es China el paradigma del nuevo crecimiento mundial, con unas tasas de crecimiento del 10\% anual en las tres últimas décadas (1980-2010). El resto de los países del entorno, incluido el subcontinente indio experimentan igualmente crecimientos significativos durante las dos últimas décadas. La última está asistiendo, además, a la dinámica emergente de determinados países de Latinoamérica, encabezados por el Brasil de Lula. Con enormes problemas, es cierto, estos cambios están, incluso, comenzando a ofrecer nuevas oportunidades a regiones mundiales que, hasta hace unas pocas décadas, habian quedado al margen de la industrialización. Nos referimos particularmente a África, parte de cuya situación queda reflejada en las tasas de crecimiento económico de los últimos años, superiores al 6\%.

En resumen, el crecimiento de la economía mundial se ha acelerado a lo largo del siglo $X X$, especialmente durante las últimas décadas. EI PIB mundial se duplicó en los primeros cincuenta años del siglo pasado, pasando de los algo más de dos billones de dólares de 1900 a los 5 billones de 1950. En 1990, ese PIB alcanzaba los casi 23 billones de dólares y, en 2007, superaba ya los 53 billones, lo que, entre 1990 y 2007, supone un ritmo medio anual de crecimiento de 1,8 billones de dólares. Este crecimiento del PIB mundial pone de manifiesto la mejora que se produce en la productividad a lo largo del siglo XX, y especialmente en su segunda mitad. El PIB per cápita mundial pasa de los 1.200 y 2.000 dólares de 1900 y 1950 respectivamente a los 8.000 de 2007. En la segunda mitad de la década de los veinte de este siglo se situará en torno a los 20.000 dólares. Pero con una precisión importante: la mayor parte de este crecimiento lo aportarán los países en vías de industrialización y los atrasados. La población definida como clases medias se habrá casi triplicado y se situará en torno a los tres mil millones. Dos terceras partes vivirán en los países actualmente en vías de industrialización y atrasados.

Sin embargo, mientras que comienza a tomar cuerpo esta nueva geografía de la industrialización mundial, el mundo occidental pierde peso de forma llamativa. Entre 1980 y 2008, el PIB de los EE.UU. y de la UE ha perdido algo más de 9 puntos porcentuales, pasando del $52 \%$ del PIB mundial en PPA (Paridad de Poder Adquisitivo) al 42,8\%, mientras que el binomio China+India ha ascendido del $4,2 \%$ al $16,2 \%$. El avance chino es especialmente espectacular, ya que pasa del $2 \%$ al $11,4 \%$. La mayor parte de los estudios (Goldman Sachs, entre otros) apuntan a que, en torno a 2025, China se habrá convertido en la primera potencia económica mundial, mientras que India se situará en un tercer o cuarto lugar. Se trata, en todo caso, de un cambio que ya se está produciendo y parece que de forma solapadamente acordada. El matrimonio económico entre la primera potencia actual y la futura queda puesto de manifiesto en las crecientes ventas de productos chinos a EE.UU., con el consiguiente importante déficit comercial. China está contribuyendo a financiar el déficit público estadounidense. Los bonos del Tesoro norteamericano de que dispone han pasado de los 50.000 millones de dólares del 2000 a cerca de los 800.000 millones de 2010. Es más que probable que, en los siguientes treinta años, China llegue a duplicar la producción de los Estados Unidos, e India se le aproxime. Los EE.UU. habrán detentado la hegemonía económica mundial por algo más de un siglo. Parece claro que, a no tardar demasiado, esa primacía retornará al "Reino del medio" para permanecer en él durante largo tiempo. Los países de la Unión Europea habrán desaparecido de entre las cinco primeras posiciones. Exceptuada la euroasiática Rusia, ningún otro país del Viejo Continente encontrará acomodo entre las cinco potencias mundiales más importantes (Goldman Sachs, 2007). 
Pero hay algo incluso más importante: el propio modelo de crecimiento basado en el uso intensivo de fuentes energéticas de origen fósil y de materias primas está llegando a su fin. El crecimiento futuro a largo plazo sólo será viable a través de una reinvención radical de modelos de crecimiento sostenibles y de sistemas de reparto más justos. Uno de los retos básicos de esta reinvención tiene que ver con el papel de la mujer. La futura economía tendrá que tomar necesariamente en valor el papel que ésta ha desempeñado durante milenios como "cuidadora de la especie", circunstancia no tenida en cuenta jamás por la ciencia económica. La integración de la mujer en todos los aspectos relacionados con la actividad humana cambiará profundamente las relaciones sociales. Y finalmente, que no en último lugar, la especie humana está obligada a replantearse muy seriamente su relación con la biosfera, ya que su alteración profunda conlleva costos irreversibles a muy largo plazo para la propia especie humana y para la diversidad biológica.

\section{2. "El Ser humano, medida de todas las cosas". La población}

En realidad, más allá del interés de la información aportada en páginas anteriores, lo realmente importante es detectar la dinámica global en que se halla envuelta la totalidad de la humanidad como especie en su esfuerzo por asegurar su propia supervivencia y la del medio que la sustenta. Hoy más que nunca, conviene no olvidar que los habitantes de esta "aldea global" conforman una misma y única especie. Es crucial profundizar en este sujeto colectivo, reflexionar sobre cómo establece sus estrategias de supervivencia, cómo se relaciona y, sobre todo, cómo se comprende a sí mismo ante los retos que cuestionan su presente y futuro. ¿Recurre siempre a idénticos mecanismos, sometida a unos patrones universales atemporales marcados biológicamente? Por lo que se puede observar en muy distintos planos, parece que, en el plazo de muy pocas generaciones, cierto que con ritmos distintos, pero más allá de imperativos nacionales, religiosos o étnicos de carácter ineludible, la humanidad está protagonizando una misma transformación adaptativa: transición demográfica y familiar, universalización educativa, modernización y cambio de parámetros económicos y políticos, urbanización, protagonismo de la mujer, etc.

\subsection{Un crecimiento demográfico acelerado y sin precedentes}

El referido a la población es el primer gran paradigma que indica que algo significativo está ocurriendo en la evolución humana. Lo expresa el aumento del número de seres humanos. En los últimos doscientos sesenta años (17502010), la población humana ha pasado de 780 a 6.500 millones de habitantes. Se ha multiplicado por ocho. Pero ese salto se atiene a un ritmo de aceleración progresivo. En los primeros ciento cincuenta años, la población se duplica. En 1900 llega a los 1.700 millones. Entre 1900 y 1950, el proceso se acelera hasta alcanzar los 2.500 millones, con un incremento del 47\%. Entre 1950 y 2010, la explosión alcanza su cenit: 6.500 millones y un incremento del $160 \%$. Algo profundamente significativo se está produciendo en el seno de la especie humana. El cambio de civilización económica (de la agraria a la industrial) y el aumento de la productividad ayudan a desvelar parte del soporte de ese crecimiento de la población. Sin embargo, esto no explica su raíz más profunda ni la de su previsible posterior estancamiento. Todo ello hay que entenderlo en el marco de un cambio radical en el modelo de régimen demográfico: la "transición demográfica".

\subsection{La "Transición Demográfica"}

Es, sobre todo, a partir de la década de los setenta del siglo pasado, cuando los estudios demográficos alcanzan un nivel de desarrollado realmente notable. De su mano, la teoría de la Transición Demográfica alcanza todo su valor como teoría explicativa de los procesos demográficos. En síntesis, la teoría viene a explicar cómo se pasa de un modelo demográfico de "viejo régimen", típico de la civilización agraria o preindustrial y caracterizado por altas tasas vitales (mortalidad, natalidad y fecundidad, y baja esperanza de vida), a otro de "nuevo régimen" basado en tasas vitales bajas y elevada esperanza de vida.

La transición de un modelo a otro se ha ido produciendo a lo largo de los últimos doscientos cincuenta años. Arranca en los países europeos de la mano de la implantación progresiva de la nueva economía industrial y del desarrollo urbano como el espacio propio en el que se produce el cambio. Dos siglos más tarde, ya en la segunda mitad del siglo XX, el crecimiento demográfico ha alcanzado dimensiones de verdadera explosión. Una parte mayoritaria de la 
humanidad se ha incorporado a él beneficiada por una doble circunstancia: la de haber traspasado definitivamente el umbral de la mortalidad del antiguo régimen, mientras que los pasos relacionados con la fecundidad y natalidad no han llegado todavía a desarrollarse en su integridad en parte importante de regiones mundiales subdesarrolladas o en vías de desarrollo. Hasta todavía recientemente, las elevadas tasas de fecundidad y natalidad características del viejo régimen seguían afectando a tres cuartas partes de la humanidad.

Sin embargo, para este momento (2010), más de la mitad de la población humana ha realizado ya su transición, lo que la lleva a instalarse dentro del nuevo modelo de equilibrio demográfico basado en bajas tasas vitales: baja mortalidad, baja fecundidad y baja natalidad. La fecundidad se sitúa en niveles inferiores a los de reproducción $(2,1$ hijos por mujer). Los datos de que disponemos actualmente (División de la Población de la ONU) señalan que, en los próximos treinta años, más del 90\% de la población humana estará totalmente inmersa en este nuevo modelo. Completadas todas las etapas de la transición demográfica, este nuevo modelo establece un nuevo régimen de estabilidad demográfica vinculado al crecimiento cero o ligeramente negativo de la población humana. De ahí que las proyecciones de la población humana para los próximos siglos hablan de una población que, hacia 2075, se situará en torno a los 9.000 millones de habitantes, para descender en la siguiente centuria y estabilizarse en esas cifras hacia el año 2300. Después de miles de años de existencia, la especie humana habrá optado por un modelo de autorregulación no comprensible desde la lógica de la sociedad en la que cobró sentido el mandato bíblico de "crecer y multiplicaos".

\subsection{Algunas pistas antropométricas}

¿Pero está cambiando tan sólo el número de los humanos y los modos de multiplicarse? El análisis de ciertas variables antropométricas señala que, durante este tiempo, la población humana ha experimentado alteraciones que afectan a su propia configuración. Nos referimos a las que se detectan en la evolución de su talla y peso.

Sabemos por múltiples estudios que, durante los últimos 150 años, el individuo de los países industriales ha incrementado sustancialmente su altura. Los datos proceden de las mediciones realizadas a los jóvenes reclutados para los ejércitos nacionales. La relación talla/peso ha permitido la construcción de un indicador adecuado para conocer la salud de una persona y su desarrollo. Es el Índice de Masa Corporal (IMC) muy utilizado en las últimas décadas por los médicos y los economistas. Permite disponer de un indicador que habla de la salud y del riesgo de morbilidad y mortalidad que tienen los individuos si traspasan, a uno $u$ otro lado del mismo, el ámbito definido como índice óptimo, y ofrece, al mismo tiempo, pistas precisas sobre el nivel de desarrollo humano de las sociedades ${ }^{3}$.

Un varón europeo de las sociedades preindustriales sería bajo y delgado. Al inicio de este período, en muchos de estos países las tallas no superaban el 1,63 metros y el indice de masa corporal se situaba por debajo del nivel 20. Ciento cincuenta años después, a finales del siglo $X X$, la talla media europea oscila entre 1,74 y 1,82 metros, de acuerdo con la situación nutricional y de desarrollo de los países respectivos, lo que equivale a un crecimiento que oscila entre 10 y 20 centímetros. Este incremento va acompañado de otro paralelo en el peso. El conjunto de estos dos indicadores señala que, en estos años, la masa corporal de los varones de los países industrializados se ha incrementado en torno a un 50\%, con un incremento paralelo de la fortaleza del individuo, de su capacidad de trabajo y de resistencia.

En las sociedades agrarias preindustriales la estructura productiva aportaba una cantidad limitada de nutrientes que situaba a los individuos en el borde nutricional mínimo vital, ligeramente por encima del consumo basal. El excedente calórico lo invertían en forma de trabajo que garantizase la producción, vital para el sostén de los individuos y de la especie. Pero lo hacía a costa de su vulnerabilidad y de aumentar el riesgo de mortalidad. El nivel nutricional y el sistema inmunológico se mantenían en niveles crónicamente críticos, lo que se traducía en una mortalidad prematura. Esto explica las altas tasas de mortalidad de estas sociedades y su baja esperanza de vida, que la especie trataba de compensar con altas tasas de fecundidad y natalidad. Cuando se Ilega a la culminación de la transición demográfica y a la consolidación del modelo de régimen demográfico de baja intensidad, la nueva situación se presenta de la mano de la imagen radicalmente renovada que ofrecen dos de las variables demográficas más significativas: alargamiento de la esperanza de vida (Eo) y baja fecundidad. 
Todo esto exige enriquecer el esquema interpretativo del crecimiento industrial de los últimos dos siglos y medio. Frente a la creencia de que la tecnología era el fundamento casi exclusivo de la industrialización, los estudios señalan que cerca del 50\% del crecimiento económico inglés de los últimos doscientos años lo aporta la mejora física de los individuos (R. W. Fogel, 2004). Y no lo olvidemos, estos cambios biofisiológicos, que no genéticos, se han producido en el plazo de seis generaciones.

\section{3. "Capital humano" y "Economía del CONOCIMIENTo". MuJer y desarRollo EDUCATIVO Y CIENTÍFICO}

Claro que ni las tasas ni los modelos demográficos cambian por sí solos, ni se explican en su integridad desde esquemas que hablan de productividad, de mejoras tecnológicas y científicas, de avances urbanísticos, higiénicos o sanitarios. Todos los citados, son, sin duda, referentes de primer orden a la hora de comprender este cambio, pero la visión que podriamos extraer del fenómeno sería radicalmente parcial si no se atendiera, con tanta o mayor solicitud, al profundo cambio cultural que paralelamente experimenta el protagonista humano, fundamentalmente la mujer. Están cambiando, de manera profunda y acelerada, los valores, los paradigmas y los referentes sociales que rigen, justifican y alientan esos comportamientos. Nos referimos a los cambios radicales que se están produciendo en la valoración del sexo y de la reproducción, en el concepto y en los modelos matrimoniales y familiares, en el papel y protagonismo de la mujer, etc. La aceleración de todo este complejo y rico proceso cuenta con dos soportes claves: la mujer y el desarrollo educativo y científico.

\subsection{El papel de la mujer en las "otras modernidades"}

La incorporación de la mujer al proceso de la modernización y concretamente al mercado del trabajo reglado está siendo muy intensa en las últimas tres décadas. Se calcula que, en este período, se han creado mil millones de puestos de trabajo, dos tercios de ellos femeninos. Los paises occidentales más avanzados están viviendo intensamente este proceso desde hace cincuenta o sesenta años; los mediterráneos, sudamericanos y asiáticos, desde algo más tarde. Esta incorporación a la actividad productiva reglada está relacionada con los efectos de la segunda transición demográfica. La "pildora" anticonceptiva constituye una de las mayores innovaciones del siglo XX. No sólo ha posibilitado el control de la natalidad, separando sexo y reproducción; ha facilitado paralelamente la creciente independencia de la mujer, poniendo a su disposición más tiempo para actividades ajenas al hogar y la crianza y una consiguiente mejora económica y educativa.

Si en las décadas finales del siglo XIX el horizonte mayoritario de la mujer estaba limitado al hogar y si, luego, en las primeras décadas del siglo $X X$, la educación primaria se le abria de manera universal, ahora lo hace a ámbitos cada vez más extensos de la educación. Esto permite a la mujer alcanzar niveles formativos equivalentes a los del varón. Ello ha tenido una influencia decisiva en la reducción del tamaño de la familia y en el cambio de las tipologías y hábitos familiares. Las mujeres que permanecen por más tiempo dentro del sistema educativo tienen menos hijos y es mayor su incorporación al mercado laboral. En los EE.UU. de 2009 trabajaba el 80\% de las mujeres con titulación universitaria y dos tercios de las que alcanzaban un nivel de enseñanza secundaria, mientras que no lo hacia el $50 \%$ de las que no poseían ningún tipo de titulación (The Economist, January, 2, 2010). Ahora mismo, a principios del siglo XXI, en los paises de la OCDE, estudian y se titulan más mujeres que hombres. Y se trata de un proceso que no sólo lo están experimentando estos países más industrializados, sino que se está extendiendo a los paises del Este y Sudeste asiático y a no pocos de América Latina.

Convendrá, en todo caso, no obviar la presencia relativamente creciente que últimamente están adquiriendo en determinados medios occidentales algunos discursos que, en sus expresiones extremas, pueden llegar a defender el retorno de la mujer a su "terreno maternal". La filósofa francesa E. Badinter denuncia este retorno forzado a un supuesto estado de naturaleza y la presión que, desde él, se ejerce sobre las mujeres para que sean madres "naturalmente" perfectas (E. Badinter, 2010). Este profundo cambio que afecta a las mismas bases estructurales del sistema no se va a producir sin resistencias. Lo pone de manifiesto la escasa presencia femenina en tres ámbitos emblemáticos del poder: el económico, especialmente en el ámbito de las grandes empresas y multinacionales, el de 
la investigación científica y el religioso de la mayor parte de las confesiones. Nos resulta llamativo que, todavia hoy, la investigación científica sea un coto extremadamente masculino. Tan sólo el 6\% de los investigadores de la OCDE son mujeres y sólo 16 de los 540 premios Nobel de ciencias. En la UE, menos de un quinto del profesorado universitario a tiempo completo es femenino.

\subsection{Alfabetización, "capital humano" y "economía del conocimiento" en la nueva globalización}

Hace ya medio siglo que la Historia Social ha venido ocupándose con especial atención del tema educativo, tanto en lo que significa como desarrollo personal, como por el peso que adquiere el "capital humano" en el proceso de modernización de las sociedades. Desde que, en 1963, se formulara la teoría de la "Transición de la alfabetización", ésta se ha convertido en instrumento analítico de primer orden a la hora de valorar los procesos de modernización y desarrollo (M. J. Bowman y C. A. Anderson, 1963). De acuerdo con ella, cada fase de industrialización lleva implícito, por lo general, el logro por parte de la población de unos niveles educativos básicos. Los diversos "umbrales de alfabetización" estarian vinculados a las distintas fases del proceso industrializador. Niveles superiores de desarrollo económico suponen y exigen la ampliación del esfuerzo docente, del tiempo educativo y de su extensión equitativa a mujeres y hombres.

Articulado en torno a un modelo cada vez más complejo, el sistema educativo se ha ido difundiendo sin solución de continuidad. Las Universidades y las Escuelas de Ingeniería se han convertido en importantes centros de creación científica y tecnológica y lo hacen, también y cada vez con mayor peso, las grandes empresas a través de sus propios departamentos de investigación y desarrollo. En el capitalismo avanzado, los estudios secundarios tienden a generalizarse y los terciarios van extendiéndose lenta, pero progresivamente a ambos géneros (entre el 30-50\% de la población de 18-24 años). La democratización está llegando también a la educación terciaria. Entre 1975 y 2000, los paises de la OCDE han visto duplicarse la proporción de población adulta con estudios superiores: del $22 \%$ al $41 \%$ (The Economist: September 2005). A lo largo del siglo XX, la ciencia se ha ido convirtiendo en la protagonista fundamental de la creación del nuevo conocimiento y de la aceleración del proceso industrial.
Esta difusión no se ha detenido en fronteras y modelos sociales. Se trata de una dinámica que, al igual que ocurría con la evolución y modernización de las estructuras productivas o con la transición demográfica, se va trasladando paulatinamente a los paises en vías de industrialización y a ritmos claramente más acelerados. Es el caso de paises emergentes como China e India. Entre 1980 y 2010, en el primero la proporción de estudiantes universitarios de entre 18 y 24 años ha pasado del 2\%-3\% al 20\% y su inversión en I+D asciende al 1,5\% del PIB. Por lo que respecta a India y parte importante de los países en vías de industrialización, parecen seguir pasos similares. Los gastos en I+D de la UE y España se sitúan respectivamente en el $1,8 \%$ y el $1,3 \%$. de sus respectivos PIB.

Resultado de este proceso y de crecientes inversiones, esta amplia base educativa se está traduciendo en un incremento notable del número de universitarios, de científicos y de producción científica, lo que está transformando profundamente la economía. En algo más de un siglo, el mundo universitario ha pasado de contar con algo más de 3 millones de individuos a superar muy ampliamente el centenar de millones. Pero conviene destacar una doble circunstancia crucial: el futuro del avance científico y, por lo tanto, del de la llamada "economía del conocimiento" va a estar estrechamente relacionado con dos aportaciones insoslayables: la de la mujer como protagonista del nuevo desarrollo y la de los nuevos paises emergentes. Los paises y regiones que no incorporen de forma temprana a la mujer a este proceso y en condiciones iguales a las del hombre perderán posiciones en los niveles de desarrollo y de bienestar.

La nueva geografía de la innovación parece que se está desplazando también, lenta pero imparablemente, hacia el Este y Sudeste de Asia. Los datos son concluyentes. China e India sacan ya al mercado un número de ingenieros superior al del mundo occidental y Japón. A día de hoy, los Estados Unidos cuentan con aproximadamente un millón y medio de científicos en los ramos de las ciencias y la ingeniería; la Unión Europea dispone de un nivel ligeramente superior y China alcanza ya esa cifra. En 2008, la producción científica mundial relacionada con las ciencias y la ingeniería alcanzó alrededor del millón y medio de artículos científicos evaluados inter pares. Un 20\% de esa producción corresponde a los Estados Unidos y China se aproxima al 10\%, frente al $2 \%$ de comienzos de los 
ochenta. Las proyecciones futuras de sus potencialidades demográficas y de desarrollo apuntan a que, en un plazo no superior a una generación, China habrá superado a Estados Unidos tanto en producción científica como en número de graduados universitarios.

Resultado de esta dinámica, para mediados del siglo en curso, en torno al $60 \%$ de la población adulta occidental

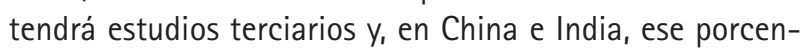
taje rondará el $40 \%$ y el $25 \%$ respectivamente. Estamos hablando de aproximadamente un tercio de la población adulta mundial, lo que hará multiplicarse exponencialmente la producción científica. Pero con dos matices: para ese momento, esa investigación se realizará en un contexto cada vez más globalizado e interconectado, uno y único, y la hegemonía del desarrollo económico se habrá desplazado al entorno del Pacífico. Como hace no más de tres siglos, ese centro retornará al extremo oriental del eje euroasiático con su extensión hacia el norte de América. La parte occidental de ese eje habrá disfrutado de su hegemonía tan sólo durante dos centurias.

El crecimiento futuro exige cambiar los "deseos" que el modelo occidental de desarrollo ha venido alimentando durante los dos últimos siglos y poner en pie un nuevo modelo sostenible sustentado en el recurso de bienes y servicios consumidores de conocimiento. En este contexto, cobrarán un protagonismo de primer orden aquellos bienes que atiendan a la salud, el ocio, el trabajo voluntario o la formación continuada (Fogel, 2004). Pero esta nueva modernidad, exige, al mismo tiempo y si no se quiere agotar en profundas divisiones y contradicciones, nuevas y más justas formas de reparto, un nuevo sistema mundial de equilibrio. Es el momento de abrirse hacia "otras modernidades" distintas de la occidental que ha regido nuestros pasos en las últimas centurias (A. Gurrutxaga, 2008).

\section{El MEdiO URBANO: LA CIUDAD, ESPACIO DE MODERNIZACIÓN}

Las "ciudades industriales", surgidas de nuevas formas productivas, renovaron el modelo urbano tradicional. Recibieron la herencia de las ciudades que las precedieron, incorporaron los nuevos mecanismos productivos, tecnológicos y organizativos derivados del desarrollo del capi- talismo y del estado-nación y conformaron el marco que hizo posible el desenvolvimiento de experiencias humanas muy distintas de las habituales en las sociedades rurales anteriores. La cercanía humana, el contacto entre personas procedentes de ámbitos distintos y la sustitución de los viejos vínculos familiares y sociales hicieron posible el desarrollo efectivo de un protagonista humano que, cada vez más alejado de los vínculos corporativos de la sociedad tradicional, fue reforzando sus perfiles más personales. Las ciudades hicieron factible la sociedad de masas, posibilitaron otras formas más democráticas de hacer política e impulsaron el movimiento obrero, la secularización o la aparición de nuevas formas de esparcimiento y de nuevos hábitos, comportamientos y valores. En tan sólo dos siglos, la población occidental rompe los vínculos con la milenaria civilización agraria. Todos los comportamientos demográficos de signo moderno, como el éxito en la batalla por el descenso de la mortalidad o el descenso de la fecundidad, encontraron su primer arraigo en las grandes ciudades. Productos y agentes de modernización, fueron percibidas como iconos de modernidad y se convirtieron en los puntos neurálgicos desde donde se difundieron las nuevas pautas sociales, las nuevas ideas y la democracia.

Los paises occidentales vivieron esta transformación en el siglo XIX y en la primera mitad del XX. Ahora bien, si durante este siglo y medio el proceso encuentra su acomodo preferente en el mundo occidental, en la actualidad, el fenómeno se ha universalizado, de manera que algunas de las mayores conurbaciones se encuentran ya fuera de ese contexto. A principios del siglo XIX, la población urbana mundial acogía el 10\%-12\% del total de la población mundial. Pertenecian a este medio 130 millones de personas. Dos siglos después, supone ya la mitad de la población mundial. Hablamos de más de 3.000 millones de personas, entre los que se cuenta el 95\% de la población occidental (UNFPA, 2008). Si, durante estos dos siglos, la población mundial se ha multiplicado por siete, la urbana lo ha hecho por veinticuatro.

El ritmo, sin embargo, no ha sido uniforme. El proceso se ha acelerado y universalizado en los cincuenta últimos años y lo continuará haciendo en el futuro. Durante este período, la mayor parte del crecimiento de la población urbana se ha producido en los paises en vías de industrialización, actualmente ya industrializados 0 en los que prosiguen por esa vía. China, el país más poblado del mundo, con 1.350 millones de habitantes, contaba, en 1980, con una 
población urbana próxima al 20\%. Un cuarto de siglo más tarde, en 2008, duplica esos porcentajes hasta llegar al 45\% (EE.UU. tardó cincuenta años en dar ese mismo salto). $\mathrm{Si}$ a finales del siglo XIX las mayores ciudades del mundo

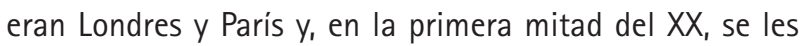
añaden Nueva York y Tokio, en los siguientes cincuenta años las megaciudades emergentes encuentran acomodo en paises de un nivel de desarrollo inferior: Shanghai, Seúl, Bombay, Yakarta, Manila, El Cairo, Lagos, México, D.F., São Paulo o Buenos Aires. Para mediados del siglo XXI, entre el $70 \%$ y $80 \%$ de la población mundial residirá en ciudades.

Las ciudades son, en definitiva, las verdaderas protagonistas del proceso de modernización que gesta la sociedad contemporánea. Lo son en el sentido más pleno y plural de ese protagonismo, que abarca desde los avances técnicos y económicos a los comportamientos demográficos, socioeducativos, políticos o culturales. Si, allá en la Baja Edad Media y entre determinados grupos sociales, cobró una cierta carta de naturaleza la apreciación de que las "ciudades olían a libertad", hoy se puede afirmar que las "ciudades industriales" son los verdaderos agentes espaciales de una modernización que, entendida como proceso, se ve obligada a mirar al futuro de manera crítica y creativa, dispuesta a reinventarse, a abrirse a "otras modernidades".

\section{Conclusiones}

La sociedad occidental actual vive, entre perpleja y angustiada, una percepción generalizada de cambio generacional profundo y acelerado. Las sensaciones de crisis, desajuste y agotamiento se instalan en el modelo como algo permanente. Los cambios e incertidumbres más visibles se hacen patentes en los ámbitos económico, social y político-administrativo. Industrialización, modernización social y desarrollo y superación posterior del marco del estado-nación hablan de un proceso de modernización nacido hace casi dos siglos y medio en el mundo occidental y que se encuentra actualmente sometido a una profunda revisión. No se puede obviar la presencia emergente de estados con más de mil millones de habitantes, como China e India, que aportan el $40 \%$ de la población humana.

Pero, más allá de estas primeras evidencias y lejos de supuestos imperativos "naturales", la especie humana viene protagonizando un largo y profundo proceso de transformación adaptativa de carácter universal. Sin embargo y medido en claves de tiempo histórico, ha sido realmente corto el camino recorrido por los humanos en el proceso de transición que les está llevando de un modelo demográfico en equilibrio, basado en altas tasas vitales y baja esperanza de vida a otro caracterizado por tasas bajas vitales y larga esperanza de vida. El nuevo modelo comporta la mejora biofisiológica y una larga longevidad. Cualquier análisis que se haga sobre el futuro habrá de tener en cuenta necesariamente estos dos aspectos. Este hombre, con larga esperanza de vida y mejores condiciones biofisiológicas, acumulará información a lo largo de su vida en un proceso de formación constante. Posiblemente su capacidad productiva e intelectual alcance su cénit en la madurez avanzada, entre los 40 y los 65 años, pero siendo muy creativo y productivo en las edades previas y posteriores. Parece que el mundo que apunta hacia un futuro de alcance medio va a caminar por estos derroteros.

Pero una lectura transversal de todos estos planos permite poner en evidencia otras tendencias de futuro que exigen igualmente ser escuchadas. El modelo de modernización de marchamo occidental basado en un crecimiento constante e ilimitado ha tocado techo. Es imposible su mantenimiento en un marco de potencialidades limitadas como las del planeta Tierra, tanto más cuanto que a las potencias tradicionales se le suma la aparición de otras emergentes de muy crecidas dimensiones. El mismo modelo de modernidad de raíz ilustrada y liberal parece haber sido traicionado por quienes lo proponían como pauta universal de desarrollo humano. Tratar de abordar retos como estos aconseja abrir nuestra reflexión occidental a otros marcos, a otras "transiciones". Es el momento de construir un nuevo modelo alternativo sostenible y más justo. Todo invita a corregir la percepción occidental que tenemos de los ritmos y de los protagonismos históricos. Occidente ha patrocinado el de los últimos siglos; los ejes de la nueva modernización parecen, sin embargo, volver nuevamente su mirada hacia el Pacífico. $Y$ ese nuevo marco ha de construirse, entre otros, sobre cuatro grandes pilares: la transformación progresiva del estatuto laboral, educativo, socio-político y cultural de la mujer, la opción decidida por la mejora rotunda de una economía intensiva en conocimiento ("capital humano"), el desarrollo equilibrado del marco físico y humano que lo hace posible, la ciudad, y un marco de gobernanza mundial. 


\section{NOTAS}

1 Un exabyte equivale a unos 10.000 millones de números de The Economist (The Economist: "Data, data everiwhere. A special report on managing information", February, 27 th., 2010).

2 Tengamos en cuenta que, cuanto más próximo se encuentre este índice del nivel 0, mayor es el grado de distribución de la riqueza y, cuanto más se acerca al nivel 100, mayor es la desigualdad.

$3 \mathrm{El}$ indicador establece la relación existente entre el peso expresado en kilogramos dividido por la talla al cuadrado expresada en metros y sus decimales. El índice óptimo se sitúa entre 22 y 25.

\section{BIBLIOGRAFÍA}

Badinter, E. (2010): Le conflit, la femme et la mère, Paris, Flammarion.

Bairoch, P. (1982): "International Industrialization Levels from 1750 to 1980", Journal of European Economic History, 11, otoño, pp. 269-334.

Bowman, M. J. y Anderson, C. A. (1963): "Concerning the role of education in development", en Geertz, C. (ed): Old societies and new states, Glencoe, The Free Press.

Bustelo, P. (2010): CHINDIA, Asia a la conquista del siglo XX, Madrid, Tecnos.

Chesnais, J. C. (1986): La Transition Démographique. Etapes, formes, implications économiques, Paris, Presses Universitaires de France, INED.

Christian, D. (2005): Mapas del Tiempo. Introducción a la "Gran Historia", BarceIona, E. Crítica.

Der Woude, A.; De Vries, J. y Hayami, A. (eds.) (1990): Urbanization in History.

Recibido: 8 de junio de 2010

Aceptado: 13 de octubre de 2010
Dyson, T.; Cassen, R. y Visaria, L. (2005): Twenty-First Century India Population. Economy, Human Development and the Environment, 0xford, Oxford University Press.

Fogel, R. W. (2000): The Fourth Great Awakening and the Future of Egalitarianism, The Chicago, University of Chicago Press.

- (2004): Escapar del hambre y de la muerte prematura, 1700-2100. Europa, América y el Tercer Mundo, Madrid, Alianza Editorial.

González Portilla, M. et al. (2001): Los orígenes de una metrópoli industrial: La Ría de Bilbao, Bilbao, Fundación BBVA.

- (2009): La consolidación de la metrópoli de la Ría de Bilbao, 2 vols., Fundación BBVA, Bilbao.

Gurrutxaga, A. (2010): Recorridos por el cambio, la innovación y la incertidumbre, Bilbao, Servicio Editorial de la UPV/EHU.

Hsieh, Ch. y Lu, M. (2004): Changing China. A Geographic Appaisal, Oxford, Westview Press.

Judt, T. (2010): Algo va mal, Madrid, Taurus.

Kao, J. (2007): Innovation Nation. How America is losing its Innovation Edge, Why it Matters, and What We Can do to Get it Back, New York, Free Press.

Livi Bacci, M. (2009): Historia Mínima de la Población Mundial, Barcelona, Ed. Crítica.

Maalouf, A. (2010): El desajuste del mundo. Cuando nuestras civilizaciones se agotan, Madrid, Alianza Editorial.

MacNeill, J. R. y McNeill, W. H. (2004): Las redes humanas. Una historia global del Mundo, Barcelona, Ed. Crítica.

Peerenboom, R. (2007): China ModernizesThreat to the West or Model for the Rest, Oxford, Oxford University Press.

Polenske, K. E. (Ed.) (2007): The Economic Geography of Innovation, Cambridge University Press. 
Robertson, R. (2005): Historia de una ciencia global, Madrid, Alianza Editorial.

Rosling, H. (2010): Entrevista de Eduard Punset con Hans Rosling, médico y estadístico del Instituto Karolinska, Suecia. Barcelona, febrero de 2010. (Vídeo del programa: http://www.redes- paralaciencia.com/2988/redes/2010/ redes-62-desmontando-mitos-sobreel-mundo).

Shapiro, R. (2009): 2020: Un nuevo paradigma. Cómo los retos del futuro cambiarán nuestro modo de vivir y trabajar, Barcelona, Ediciones Urano.
Touraine, A. (1993): Crítica de la Modernidad, Madrid, Temas de Hoy, S. A.

Xizhe, P. y Zhigang, G. (eds.) (2000): The Changing Population of China, Madrid, Blackwell Publishers.

Zakaria, F. (2009): El mundo después de USA, Madrid, Espasa Calpe. 